\title{
Evaluation Research on Emergency Management Capability of College Accidents Based on Improved LM-RBF Neural Network
}

\author{
Ning Cheng \\ School of Economics and Management \\ Beihang University \\ Beijing, China
}

\author{
Xiaodong Song* \\ School of Economics and Management, \\ Beihang University \\ Beijing, China \\ 09395@buaa.edu.cn
}

\begin{abstract}
College emergency management is an important approach to maintain order and secure safety of campus. For colleges, setting up a scientific and effective evaluation model of emergency management capability is not only an important means to enhance the level of emergency management, but also the key to ensure normal operation of education. This paper proposes an improved RBF artificial neural network algorithm based on LM. This algorithm improves the compact ratio and error convergence speed of RBF neural network, and has better processing ability and higher robustness in the highly nonlinear problem-emergencies.
\end{abstract}

Keywords-Emergency management capability; RBF neural network; LM error correction algorithm

\section{INTRODUCTION}

Due to social changes and the development of Internet technology, colleges and universities gradually have evolved from a relatively closed system to a relatively open system which gives them an effective communication access to the society. All kinds of information in the society infiltrates into universities through various means such as the Internet and media, which brings many instability factors to the daily management of colleges. Therefore, the establishment of a scientific evaluation model of emergency management capability can improve college emergency management capability.

\section{PROPOSED APPROACH}

\section{A. Basic RBF-Artificial Neural Network}

Traditional RBF neural network construction selects core neurons by using K-means, Kohonen algorithm

and so on, and then selects a core neuron training algorithm to generate RBF neurons with fixed width and height. This kind of algorithm can obtain the RBF neural network with better generalization ability [1]. The basic topological structure is shown in Fig. 1.

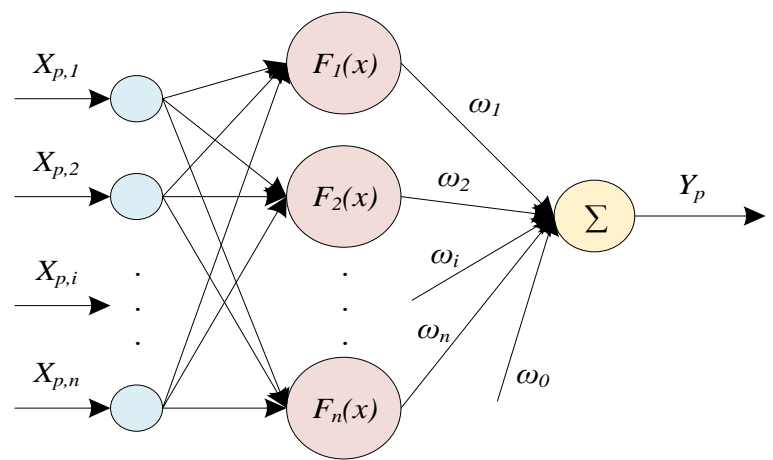

Fig. 1. RBF neural network topology.

Fig. 1 is a standard three-layer RBF neural network, where F represents the kernel function, usually Gaussian function. For any sample $p$, the kernel function of the h-th RBF neuron is shown in (1):

$$
F_{h}\left(x_{p}\right)=e^{-\frac{\left\|x_{p}-c_{h}\right\|^{2}}{\sigma_{h}}}
$$

Equation (1) includes two parameters, namely $c_{h}$ and $\sigma_{h}$, represent the center and width of the neuron respectively, and $\|-\|$ is the Euclidean distance.

\section{B. Improved LM Error Correction Algorithm}

Levenberg-Marquardt algorithm is a widely used non-linear least squares method in neural networks training and other optimization problems. However, the traditional LM algorithm needs Jacobian matrix multiplication, and the solution process takes up a lot of space which is difficult to be solved under the existing hardware conditions. Therefore, an improved LM algorithm is proposed to solve this problem. The improved algorithm is illustrated below:

Any vector $\boldsymbol{j}_{\boldsymbol{i}, \boldsymbol{k}}$ in the Jacobian matrix can be expressed as (2): 


$$
j_{i, k}=\left[\frac{\partial_{e_{i, k}}}{\partial_{\Delta_{n, 1}}}, \frac{\partial_{e_{i, k}}}{\partial_{\Delta_{n, 2}}}, \ldots, \frac{\partial_{e_{i, k}}}{\partial_{\Delta_{n, H}}}\right]
$$

Then each quasi-Hessian submatrices $\boldsymbol{q}$ is calculated as (3):

$$
q_{i . k}=j_{i, k}^{T} j_{i, k}
$$

So, the quasi-Hessian matrix $\boldsymbol{Q}$ consists by q is in (4):

$$
Q=\sum_{i=1}^{p} \sum_{k=1}^{N} q_{i, k}
$$

Similarly, the gradient vector $\boldsymbol{g}$ is shown in (5):

$$
g=j_{i, k} e_{i, k}
$$

Here, the method of calculating the quasi-Hessian matrix $\boldsymbol{Q}$ is the same as the traditional LM algorithm, but it does not need to calculate and store the entire Jacobian matrix. Instead, it only calculates one row vector in the Jacobian matrix every time.

\section{RBF Neural Network Training Algorithm Based on the Improved LM Error Correction Algorithm}

According to the improved LM algorithm, the update rule of network parameters is:

$$
\Delta_{n+1}=\Delta_{n}-\left(Q_{n}+\mu_{n} I\right)^{-1} g_{n}
$$

In (6), the set $\Delta$ containing all the adjusting parameters can be written as $\left\{c_{n}, \sigma_{n}, \omega_{n}\right\}$, where $\boldsymbol{c}$ is the center, $\boldsymbol{\sigma}$ is the width and $\boldsymbol{\omega}$ is the weight. $\boldsymbol{n}$ represents the iterations, $\boldsymbol{Q}$ is the quasiHessian matrix, $\boldsymbol{I}$ is a unit matrix, $\boldsymbol{\mu}$ is a combination coefficient, and $\boldsymbol{g}$ is a gradient vector.

$\boldsymbol{Q}$ can be obtained by adding quasi-Hessian submatrices $\boldsymbol{q}$, as shown in (7).

$$
Q=\sum_{p=1}^{P} q_{p}, q_{p}=j_{p}^{T} j_{p}
$$

Gradient vector $\boldsymbol{g}$ can be obtained by adding subgradient vectors $\eta_{p}$, as shown in (8).

$$
g=\sum_{p=1}^{P} \eta_{p}, \eta_{p}=j_{p}^{T} e_{p}
$$

$\boldsymbol{e}_{p}$ is the difference between the expected output and the actual output.

\section{EXPERIMENTS}

\section{A. Index System Construction}

The establishment of a complete, comprehensive and scientific index system is the prerequisite to evaluate the emergency management capability in colleges and universities. This paper builds up the evaluation index system based on the 4R Crisis management theory so as to enhance the systematic and scientific index system. 4R Crisis management theory divides emergency management into four stages-readiness, response, recovery and reduction.

Based on the 4R Crisis management theory, combining the new situation and features of emergency management in colleges and universities within the Internet, this paper constructs the evaluation index system of college emergency management capability from four aspects: basic guarantee, precaution, execution and feedback. The index system is shown in Table I.

\section{B. Data Collection and Preprocessing}

50 colleges and universities all over the country as samples were selected for this research. The survey adopted stratified sampling principle and took the difference of the university levels as the stratification criterion.

\section{1) Result analysis}

a) Training of the RBF neural network: We selected ten universities randomly as testing samples, considered the other universities as training samples, and the Matlab 2014b is utilized to construct the network. The optimum value of the number of nodes in hidden layers and the spread constants are obtained through continuous practice and comparison, and the optimal results are 5.5 and 9.0 respectively. From the training results we can see that the emergency management capability of 23 universities out of the training sets seems well while the emergency management capability of the other 17 universities is modest. In the testing sets, 4 universities perform well while the other 6 universities reach relatively fair performances.

b) Analysis of discriminant accuracy of the model: The comparison of discriminant results of samples using different algorithms including improved LM-RBFANN algorithm, improved GM algorithm ${ }^{1}$, SVM algorithm and logit algorithm are shown in Fig. 2. The result proves that the university emergency management model constructed in our paper has a good discriminant accuracy and discriminant effect. 


\section{:TUNVVLUNV

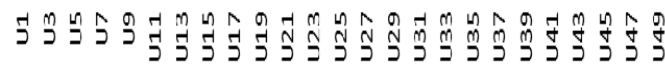 \\ :几NNVULWNV}

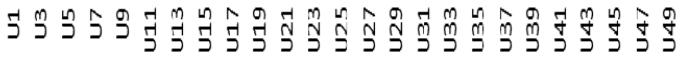

$\begin{array}{ll}\text { (a) LM-RBFANN } & \text { (b) GM }\end{array}$
רWWNVWMUNM

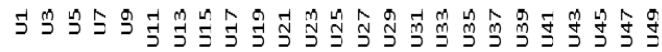

WWMN LMMNN

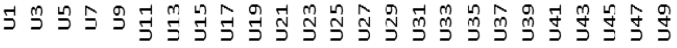

(c) SVM

(d) Logit

a. Note: In Fig 2, 1 indicates that the emergency management capability is well, while 0 is modest.

Fig. 2. Result of different algorithms.

TABLE I. EVALUATION INDEX SYSTEM OF COLLEGE EMERGENCY MANAGEMENT CAPABILITY

\begin{tabular}{|c|c|c|}
\hline First Grade Index & Second Grade Index & Measure Index \\
\hline \multirow{10}{*}{ Basic Guarantee } & \multirow{3}{*}{$\begin{array}{l}\text { Professional } \\
\text { Investment }\end{array}$} & Ratio of Teachers \\
\hline & & Ratio of Senior Professional Teachers \\
\hline & & Ratio of Emergency Management Staff \\
\hline & \multirow{2}{*}{ Network Staff Investment } & Ratio of Emergency Internet Management Staff \\
\hline & & Ratio of Network Emergency Management Researchers \\
\hline & \multirow{2}{*}{ Device Investment } & Ratio of Daily Emergency Management Investment \\
\hline & & Funding Investment Ratio of Network Security Equipment \\
\hline & Fund Investment & Amount of Fund \\
\hline & \multirow{2}{*}{$\begin{array}{l}\text { Construction } \\
\text { Emergency Center }\end{array}$} & Completeness Degree of the Organization Structure \\
\hline & & Construction of Network Emergency Center \\
\hline \multirow{11}{*}{ Precaution } & \multirow{2}{*}{ General Plan Construction } & Amount of Emergency Management Plan \\
\hline & & Feasibility of Emergency Management Plan Implementation \\
\hline & \multirow{3}{*}{ Network Supervision } & Amount of Network Plan \\
\hline & & Coverage Degree of Network Supervision \\
\hline & & Feasibility of Network Plan \\
\hline & \multirow{3}{*}{ Safety Education } & Number of Annual Safety Education \\
\hline & & Coverage of Safety Education \\
\hline & & Number of Emergency Exercises \\
\hline & \multirow{3}{*}{$\begin{array}{l}\text { Popularity of Network } \\
\text { Security Knowledge }\end{array}$} & Number of Annual Network Security Education \\
\hline & & Number of Annual Network Security Practice \\
\hline & & Coverage of Network Security Course \\
\hline \multirow{6}{*}{ Execution } & \multirow{2}{*}{ Execution Efficiency } & Start Efficiency of Emergency Plan \\
\hline & & Execution Process of Emergency Plan \\
\hline & Execution Effect & Execution Effect of Existing Emergency Plan \\
\hline & \multirow{2}{*}{ Network Control } & Effect of Public Internet Opinion Control \\
\hline & & Effect of Public Internet Opinion Soothing \\
\hline & Emergency Ability & College Emergency Disposal \\
\hline \multirow{7}{*}{ Feedback } & \multirow{3}{*}{ Learning Ability } & Correction of Problem Plan \\
\hline & & Update of Existing Plan \\
\hline & & Research on Excellent Plan \\
\hline & Emergency & Students' Satisfaction of Emergency Management \\
\hline & Management & Teachers' Satisfaction of Emergency Management \\
\hline & Southin A hiliti & Soothing Ratio After the Emergency \\
\hline & Soothing Ability & Students' Satisfaction After the Emergency \\
\hline
\end{tabular}




\section{CONCLUSION}

Based on the improved LM-RBF artificial neural network, this paper constructs an emergency management capability evaluation model for colleges and universities. The results show that this model has relatively good discriminant effect and can solve the problem of discriminant neural networks with high dimensions in large samples, which has high promotional value.

\section{REFERENCES}

[1] J. Liu, P. Yang, W.S. Lu, and A. Liu, "BP and RBF Artificial Neural Network Modeling and Classified Evaluation for Urban Air Quality," Safety and Environmental Engineering, vol. 21(6), pp. 129-134, 2014. 